\title{
Developmental Changes in Diffusion Anisotropy Coincide with Immature Oligodendrocyte Progression and Maturation of Compound Action Potential
}

\author{
Alexander Drobyshevsky, ${ }^{1}$ Sheng-Kwei Song, ${ }^{4}$ Georgi Gamkrelidze, ${ }^{2}$ Alice M. Wyrwicz, ${ }^{3}$ Matthew Derrick, ${ }^{1}$ Fan Meng, ${ }^{1}$ \\ Limin Li, ${ }^{3}$ Xinhai Ji, ${ }^{1}$ Barbara Trommer, ${ }^{2}$ Douglas J. Beardsley, ${ }^{5}$ Ning Ling Luo, ${ }^{5}$ Stephen A. Back, ${ }^{5 *}$ and \\ Sidhartha $\operatorname{Tan}^{1 \star}$ \\ Departments of ${ }^{1}$ Pediatrics and ${ }^{2}$ Neurology and ${ }^{3}$ Center for Basic MR Research, Northwestern University and Evanston Northwestern Healthcare, \\ Evanston, Illinois 60201, ${ }^{4}$ Department of Radiology, Washington University, St. Louis, Missouri 63110, and ${ }^{5}$ Department of Pediatrics, Oregon Health \\ Science University, Portland, Oregon 97201
}

Disruption of oligodendrocyte lineage progression is implicated in the white-matter injury that occurs in cerebral palsy. We have previously published a model in rabbits consistent with cerebral palsy. Little is known of normal white-matter development in perinatal rabbits. Using a multidimensional approach, we defined the relationship of oligodendrocyte lineage progression and functional maturation of axons to structural development of selected cerebral white-matter tracts as determined by diffusion tensor imaging (DTI). Immunohistochemical studies showed that late oligodendrocyte progenitors appear at gestational age 22 [embryonic day 22 (E22)], whereas immature oligodendrocytes appear at E25, and both increase rapidly with time $\left(\sim 13 \mathrm{cells} / \mathrm{mm}^{2} / \mathrm{d}\right)$ until the onset of myelination. Myelination began at postnatal day 5 (P5) (E36) in the internal capsule (IC) and at P11 in the medial corpus callosum (CC), as determined by localization of sodium channels and myelin basic protein. DTI of the CC and IC showed that fractional anisotropy (FA) increased rapidly between E25 and P1 (E32) $(\sim 11 \%$ per day) and plateaued ( $<5 \%$ per day) after the onset of myelination. Postnatal maturation of the compound action potential (CAP) showed a developmental pattern similar to FA, with a rapid rise between E29 and P5 (in the CC, $18 \%$ per day) and a slower rise from $\mathrm{P} 5$ to $\mathrm{P} 11$ (in the $\mathrm{CC},<5 \%$ per day). The development of immature oligodendrocytes after E29 coincides with changes in FA and CAP area in both the CC and IC. These findings suggest that developmental expansion of immature oligodendrocytes during the premyelination period may be important in defining structural and functional maturation of the white matter.

Key words: brain development; diffusion tensor imaging; sodium channel; compound action potential; conduction velocity; oligodendrocyte

\section{Introduction}

We previously developed a rabbit model of preterm hypoxiaischemia that manifests early postnatal hypertonic motor and neurosensory deficits suggestive of cerebral palsy (Derrick et al., 2004). There is considerable evidence supporting a central role for white-matter involvement in the pathogenesis of cerebral palsy (Volpe, 2001; Back and Rivkees, 2004). White-matter injury is often observed in preterm babies, the most vulnerable population for cerebral palsy, and is exemplified in periventricular leucomalacia. White-matter damage has been ascribed to maturational factors that precede the onset of myelination. These

Received Dec. 7, 2004; revised March 24, 2005; accepted May 8, 2005

This work was supported by National Institutes of Health Grants NS43285, NS41476, 1 S10 RR15685-01, NMSS RG 3376-A-2/1, and CA 1012-A-13.

*S.A.B. and S.T. are joint senior authors.

Correspondence should be addressed to Dr. Alexander Drobyshevsky, Department of Pediatrics, Northwestern University and Evanston Northwestern Healthcare, 2650 Ridge Avenue, Evanston, IL 60201. E-mail: oldrobys@northwestern.edu.

D01:10.1523/JNEUROSCI.4983-04.2005

Copyright $\odot 2005$ Society for Neuroscience $\quad$ 0270-6474/05/255988-10\$15.00/0 include maturation-dependent vulnerability of the disruption in oligodendrocyte lineage (Back et al., 2002) and possibly axons (Dammann et al., 2001).

We used three different systemic approaches to define the developmental trajectory of rabbit white-matter development. The first was diffusion tensor imaging (DTI) by magnetic resonance imaging (MRI) that is increasingly being used as a noninvasive diagnostic marker for perinatal brain injury. Among the DTI parameters, the progressive maturation of white matter can be assessed by diffusion anisotropy as quantified by fractional anisotropy (FA), an orientation-independent parameter (Klingberg et al., 1999). DTI has been used to assess white-matter and gray-matter development before and after the onset of myelination in animals (Mori et al., 2001; Prayer et al., 2001; Zhang et al., 2003) and human neonates (Neil et al., 1998). Abnormal progression of DTI parameters is associated with perinatal injury, and it is being increasingly used to study human brain injury (Huppi and Inder, 2001; Miller et al., 2002; Mirmiran et al., 2004).

Because the cellular basis of diffusion anisotropy is unresolved, we developed functional and histological correlates of 
DTI parameters of relevance to normal CNS development and perinatal brain injury. Functional maturation and injury of white matter has been defined by the progression of the compound action potential (CAP) magnitude and conduction velocity (CV) in mammals (Rasband et al., 1999; Tekkok and Goldberg, 2001; Baker et al., 2002; Cabanes et al., 2002), and we adapted these techniques as our second modality.

Third, we defined the developmental progression of late oligodendrocyte progenitors and immature oligodendrocytes, to better define cellular mechanisms related to the maturation of structural (defined by diffusion anisotropy) and functional elements (defined by CAP and CV) in normal white matter. We tested the hypothesis that diffusion anisotropy is related to the timing of oligodendrocyte lineage progression and functional maturation of axons. Because myelination results in redistribution of sodium channels $(\mathrm{NaCh})$ with clustering at the nodes of Ranvier (Rasband et al., 1999), we also used this approach to quantify the status of myelination as the final stage in oligodendrocyte lineage.

We found that maturation of diffusion anisotropy in perinatal development coincides with the timing of appearance of immature oligodendrocytes before myelination as well as CAP area. CV increased initially at the same rate as anisotropy, but with the onset of myelination, the rate of $\mathrm{CV}$ growth was much more rapid.

\section{Materials and Methods \\ Animal preparation}

Pregnant New Zealand White rabbit dams were allowed to give birth naturally at $31.5 \mathrm{~d}$ after conception [embryonic day 31.5 (E31.5)]. The rabbit pups were kept with the dams and underwent in vivo MRI at various ages. Pups at the ages postnatal day 1 (P1) (E32), P5 (E36), P8 (E39), and P11 (E42) ( $n=7-9$ per age group) were sedated with an intramuscular injection of a mixture of ketamine $(35 \mathrm{mg} / \mathrm{kg})$, xylazine ( 5 $\mathrm{mg} / \mathrm{kg}$ ), and acepromazine ( $1 \mathrm{mg} / \mathrm{kg})$ for MRI. Because a rodent stereotaxic device was not suitable for the rabbit pups, considering the soft skull bones, small front teeth, and closed external ear canals, we used a special setup for MRI to reduce motion artifacts. The pups were placed in a supine position in a custom-made cradle, the head was rested on a mold with the surface coil, and the upper jaw was fixed with elastic string. In this position, the immobilized head of the pup was slightly elevated from the rest of the body, reducing respiratory motion artifacts. The animals were kept warm with a heated water blanket at $35^{\circ} \mathrm{C}$, and a rectal temperature probe was used to monitor body temperature. The respiratory rate was monitored using an adhesive pressure sensor attached to the pup's chest. Temperature and respiration sensors were connected to an MP150 physiological monitoring system (BioPack Systems, Goleta, CA).

Another set of pups at postnatal ages P1, P5, and P11 ( $n=5-6$ per age group) was killed after inhalation of isoflurane anesthetic and decapitated, and the brains were obtained for electrophysiology.

In a separate set of dams, a hysterotomy was performed at E22, E25, and E29, and fetal brains were extirpated. Brains were immersion fixed with $4 \%$ paraformaldehyde and underwent ex vivo DTI ( $n=6$ per age group). Brains from $\mathrm{P} 1$ rabbits that were scanned in vivo $(n=4)$ were immersion fixed with $4 \%$ paraformaldehyde and underwent a second DTI scan ex vivo.

\section{MRI methods}

MRI was performed on a 4.7 T Bruker (Billerica, MA) Biospec system interfaced with a horizontal magnet, running ParaVision 3.0 software (Bruker), with custom-made surface coils used for excitation and reception [inner diameter, $28 \mathrm{~mm}$ (P1, P5, and P8) or $35 \mathrm{~mm}$ (P11)]. After acquiring a multislice sagittal localizer scan, DTI experiments were performed using the Stejskal-Tanner spin-echo diffusion-weighted sequence: $\delta=5 \mathrm{~ms} ; \Delta=15 \mathrm{~ms}$; repetition time (TR)/echo time (TE), 2000/35 ms; six images with noncollinear diffusion weighting with $\mathrm{b}=$ $780 \mathrm{~s} / \mathrm{mm}^{2}$ and one reference image with no diffusion weighting; eight averages were taken. The images were visually inspected, and if noticeable motion artifacts were detected, then these images were discarded a priori to analysis. Ten oblique coronal brain slices were acquired; the matrix size was $128 \times 64$, zero-filled to $128 \times 128$. The slice thickness/in-plane resolution after interpolation was $1 / 0.156 \mathrm{~mm}$ for $\mathrm{P} 1,1.2 / 0.179 \mathrm{~mm}$ for P5, 1.3/0.179 mm for P8, and 1.5/0.195 mm for P11 pups. Slice thickness was chosen to keep the number of slices constant and cover approximately the same area of cerebrum from the posterior edge of olfactory bulbs to the anterior edge of the superior colliculus for all age groups. This was done to ensure that the image planes were approximately the same for all ages, both for in vivo and ex vivo scans and for histology and electrophysiological measurements on the corpus callosum.

The fixed brains were examined using a $4.7 \mathrm{~T}$ magnet interfaced with a Varian (Palo Alto, CA) Unity-Inova console. The acquisition parameters were as follows: TR/TE, 2500/38 ms; b $=0$ and $1815 \mathrm{~s} / \mathrm{mm}^{2}$; matrix, $128 \times 128$; field of view (FOV), $2 \mathrm{~cm}$; slice thickness, $0.5 \mathrm{~mm}$; 12 averages. Diffusion times were chosen to match the mean diffusion distance with characteristic dimensions of brain tissue and were kept the same for in vivo and ex vivo measurements.

The b-values were chosen to get optimal signal attenuation, determined by the product of the apparent diffusion coefficient (ADC) and b-value, in the range of $35-65 \%$ for a robust estimation of the diffusion tensor. The ADC values of white-matter structures, estimated from pilot scans in different white-matter tracts including the internal capsule and corpus callosum, were $1.25 \times 10^{-3} \mathrm{~mm}^{2} / \mathrm{s}$ for in vivo samples and $0.18 \times$ $10^{-3} \mathrm{~mm}^{2} / \mathrm{s}$ for ex vivo samples. The mean values of the ADC of the selected tracts were weighted by the number of voxels in the regions of interest (ROIs).

Diffusion tensor was calculated using multivariant linear fitting of signal attenuation from the acquired diffusion-weighted images (Basser and Jones, 2002). Principal eigenvectors and eigenvalues were obtained after diffusion tensor diagonalization. The ADC, axial (first eigenvalue of the diffusion tensor) and radial (average of the second and third eigenvalues) diffusivities (Song et al., 2002), and FA index maps were calculated (Basser et al., 1994) using in-house software written in Matlab 5.6 (MathWorks, Natick, MA). Directionally encoded FA maps were used to identify major fiber tracts and selected ROIs (see Fig. $5 \mathrm{D}$ ). In the colorcoded image, the red, green, and blue value of each pixel was defined by the direction of its primary eigenvector, and intensity was proportional to FA (Pajevic and Pierpaoli, 1999). Red, green, and blue colors corresponded to fiber orientation in left-right, dorsoventral, and craniocaudal orientation, respectively.

The T2-weighted spin-echo sequence was performed with the same slice position and geometry parameters with a TR of $4000 \mathrm{~ms}$. T2 parametric maps were obtained by fitting a monoexponential signal decay curve from eight spin-echo images with echo times varying from 20 to $160 \mathrm{~ms}$.

\section{ROI segmentation}

Automated segmentation. To minimize observer variability and bias, an automated region-outlining routine, similar to previously published methods (Sun et al., 2003a; Klose et al., 2004), was written in Matlab to delineate the regions of the corpus callosum and internal capsule. A single seeding voxel was selected manually by clicking on the structure of interest. An iterative procedure using FA and an angle between primary eigenvectors of a seed voxel and neighboring voxels, as determined by their inner product, was used for region growing. If the angle and FA value of the voxel in question fell within the predefined threshold, this voxel was assigned to the ROI and became the seed point for the next iteration. The procedure let the ROI grow to the adjacent slices, where the structure of interest was present. Special rigidity constraints, implemented as morphological opening on each iteration step, prevented erroneous ROI leaking to the adjacent structures and ensured a smooth shape of the ROI. The cutoff value of 0.30 for FA and 0.94 for the inner product between primary eigenvectors for neighboring voxels was chosen because it allowed robust segmentation of the corpus callosum and internal capsule of perinatal rabbits. The obtained ROIs were always checked on anatomical images and corrected manually, if necessary. The coefficient of variation of the number of voxels at $\mathrm{P} 1$ and $\mathrm{P} 11$ was 7.8 and 


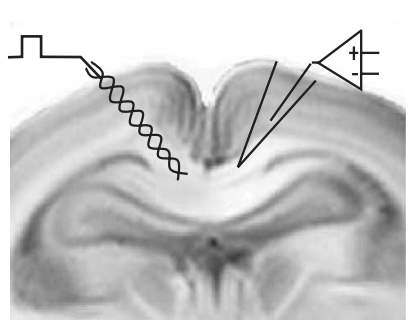

A

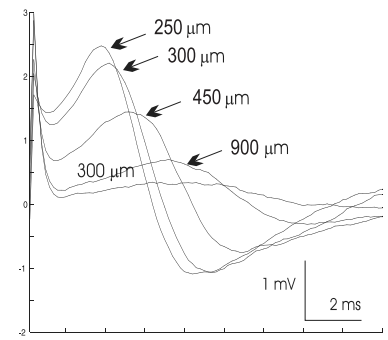

B

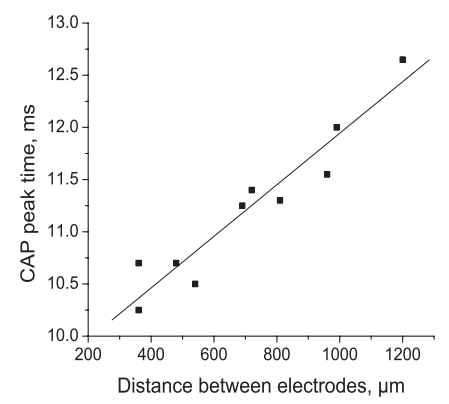

C

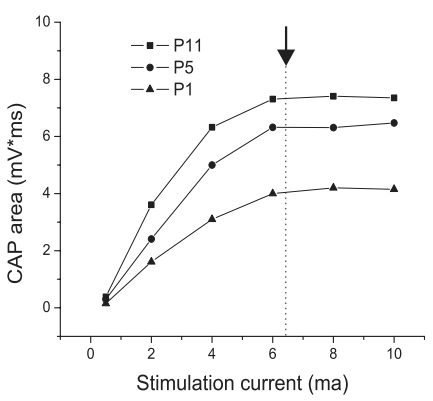

$\mathrm{D}$

Figure 1. A, The CAP was measured in the corpus callosum using stimulating (left) and recording (right) electrodes. $\boldsymbol{B}$, The onset time of the first positive peak and area under the curves was measured at various distances between electrodes (250-900 $\mu \mathrm{m}$ ). C, The onset of the CAP (ordinate) was plotted to the distance between electrodes (abscissa). Linear regression was significant $(p<0.05)$ with $R^{2}=0.98$. The CV of the CAP was determined as the slope of the line depicted. $\boldsymbol{D}$, The amplitude and area of the CAP increased proportionally to the stimulation intensity and saturated at certain threshold levels for each age. A step current stimulation was used, and measurements of CV and CAP area were finally done at a suprathreshhold level (arrow).

$11.3 \%$ for the corpus callosum and 12.5 and $13.8 \%$ for the internal capsule, respectively.

Manual segmentation. Structures with no preferred directionality and low FA values were outlined manually by placing polygon ROIs using Matlab. White-matter ROIs, such as the external capsule and corona radiata, were outlined on a color directionally encoded FA map, which greatly facilitated detection of the fiber tracts. Detected white-matter tracts served as references in the placement of the ROI for gray matter in the cortex, hippocampus, putamen, and caudate nucleus. ROIs were places on all slices where anatomical structures of interest were present.

Values of the diffusion indices for a given ROI, drawn on several slices, were calculated as an average for all of the voxels in the ROI.

\section{Electrophysiology methods}

The entire brain of rabbit fetuses or pups $(n=3-5$ per tract per age group) was quickly dissected, placed into ice-cold artificial CSF (ACSF), and oxygenated with $95 \% \mathrm{O}_{2}-5 \% \mathrm{CO}_{2}$ at $\mathrm{pH}$ 7.4. The ACSF consisted of the following (in mM): $124 \mathrm{NaCl}, 3 \mathrm{KCl}, 2.4 \mathrm{CaCl}_{2}, 1.3 \mathrm{MgSO}_{4}, 1.25$ $\mathrm{NaH}_{2} \mathrm{PO}_{4}, 26 \mathrm{NaHCO}_{3}$, and 10 glucose. Coronal slices, containing the corpus callosum, and horizontal slices, containing the internal capsule, were cut at a thickness of $500 \mu \mathrm{m}$ with a vibrating tissue slicer (Vibratome Series 1000; Vibratome, St. Louis, MO) and allowed to recover in oxygenated ACSF at room temperature for 45-60 min before recording. The slices were transferred as needed to a perfusion chamber, oxygenated with $95 \% \mathrm{O}_{2}-5 \% \mathrm{CO}_{2}$, and maintained at $21^{\circ} \mathrm{C}$ in ACSF. A bipolar stimulating electrode (constructed of twisted Teflon-coated platinum wire; diameter, $0.025 \mathrm{~mm}$ ) and a recording electrode (glass capillary extracellular microelectrode) were inserted in the middle third of the corpus callosum along the fiber course (Fig. 1A). The internal capsule was measured in a similar manner on horizontal slices obtained from a separate set of animals.

The CAP was evoked using current steps with a duration of $50 \mu$ s and an amplitude $6.5 \mathrm{~mA}$. The stimulation amplitude was selected to be $\sim 10 \%$ above the level required for a maximum response for all age groups (Fig. 1D). Recorded CAPs were amplified 1000 times, digitized at $8 \mathrm{kHz}$, bandpass filtered $(0.3 \mathrm{~Hz}$ to $10 \mathrm{kHz})$, and acquired using pClamp 8.1 software (Molecular Devices, Union City, CA). The electrophysiological properties that were measured in the CAP were threshold-to-evoked maximum response, positive peak time and amplitude, and CAP CV. The CV of the CAP was calculated as the slope of the linear fit of the distance between electrodes and CAP arrival time, as determined by the peak of the fastest positive component of the CAP (Fig. $1 \mathrm{~B}$ ). The distance between electrodes was varied from 150 to $1500 \mu \mathrm{m}$, measured by a reticle placed in a microscope eyepiece. The CAP was registered at 8-14 data locations in the fiber tract per slice, two or more slices per animal. The quality of the fit was checked by regression with $R^{2}$ always $>0.9$ (Fig. $1 C)$. The amplitude of the CAP rapidly decreased with increasing distance between the electrodes. The measurements of stimulus current to evoke maximum CAP amplitude and area were standardized across an- imals by using a fixed interelectrode distance, arbitrarily chosen as 700 $\mu \mathrm{m}$ for the nonmyelinated fibers with slow CV. At this relatively small distance, the CAP was readily separated from the stimulus artifact and, at the same time, could be robustly evoked with high amplitudes, because the likelihood of placing electrodes on the same fiber course was high. For calculating the CAP area, the baseline before stimulation was set to zero and the area was measured by integrating absolute values of the CAP for $5 \mathrm{~ms}$ from the end of the stimulation artifact. Integration was done using a custom-made routine in Matlab.

\section{Immunohistochemistry}

A detailed method for $\mathrm{NaCh}$ immunostaining has been described previously (Rasband et al., 1999). Briefly, freshly excised brains were cut in 5-mm-thick slices just rostral to bregma and corresponded to the fourth MRI slice containing the splenium of the corpus callosum. The slices were frozen in OCT compound, and $6 \mu \mathrm{m}$ sections were cut on a Leica (Bannockburn, IL) CM 1850 cryostat. Slides were then fixed in methanol and acetone (v/v 50:50), washed, and incubated in 3\% hydrogen peroxide and methanol. After washing and incubating with blocking buffer ( $2 \%$ BSA and $5 \%$ goat serum), slices were incubated with a pan-speciesspecific and pan-isoform-specific monoclonal antibody against voltagegated NaCh (mouse anti- NaCh IgG; catalog \#S8809; 1:200; Sigma, St. Louis, MO) and then with an HRP-labeled secondary antibody (G-21040; Molecular Probes, Eugene, OR). Preparations were examined by fluorescence-deconvolution digital imaging with a Leica (Wetzlar, Germany) DMRX upright fluorescent microscope. The NaCh clusters that form at the nodes of Ranvier along the axons were visible as bright puncta on myelinated axons as opposed to diffuse staining during the premyelination phase. The number of $\mathrm{NaCh}$ clusters was counted in a $100 \mu \mathrm{m}^{2}$ FOV. An average of six FOV counts from two adjacent slices in the corpus callosum and in the internal capsule was taken.

\section{Quantitative morphometric analysis of 04 - and O1-labeled cells} Immunohistochemical localization of the $\mathrm{O} 4, \mathrm{O} 1$, and anti-myelin basic protein (MBP) antibodies was performed as reported previously (Back et al., 2002). In each animal, cell counts were obtained from a minimum of five pairs of 50- $\mu \mathrm{m}$-thick adjacent sections that were alternately stained with the $\mathrm{O} 4$ or $\mathrm{O} 1$ antibody. Briefly, the nucleus was selected as the smallest countable object and was visualized by immunofluorescent counterstain with Hoechst 33324. Cell profiles that contained a nucleus were counted with a $40 \times$ objective equipped with a counting grid that was mounted on a Leica DMRA upright fluorescent microscope. Cell counts were obtained from no less than 20 high-power fields. Cells were systematically counted in similar sectors of adjacent sections. At P2 or P7, 15 high-power fields were analyzed in the corpus callosum (the region sampled extended laterally from the medial corpus callosum at the level of the sagittal sulcus).

To ensure that the same region of the corpus callosum and internal capsule was analyzed in each animal, brains were blocked in a matrix 
tissue slicer to define the true coronal plane. Serial sections were collected from the anterior tips of the olfactory bulbs through the hippocampal formation. Cell counts were done in sections from one standardized region that began at the anterior extent of the fimbria of the hippocampus where the columns of the fornix and the anterior thalamic nuclear group are present. The presence of these structures was verified by fluorescent counterstain of the tissue sections with the nuclear dye Hoechst 33324.

\section{Coregistration of the data sets}

Because the stereotaxic atlas of the newborn rabbit pup was not available and the rabbit pup brain is unlike that of the rodent pup (does not have a flat dorsal surface), we could not transfer rodent stereotaxic positioning methods to the rabbit. Our method of coregistration was as follows.

To ensure that oblique imaging sections were parallel to the plane used for ex vivo imaging, slices were placed perpendicular to the plane connecting the two most inferior points of the cerebrum, as determined with the aid of a multislice sagittal localizer scan. Similar slicing planes were obtained on the excised brain, placed on the flat surface, and sectioned perpendicular to this surface. Slices for acute electrophysiological measurement on the corpus callosum were made in this manner at the level of midpoint of the fissura sagittalis lateralis, which was identifiable on the brain surface and MRI cross sections (level of hippocampus and anterior thalamic nuclei, $0.5-1 \mathrm{~mm}$ posterior to bregma). The landmarks used were bregma, midpoint of the fissura sagittalis lateralis, and the most inferior parts of the cortex. Visual comparison showed that the histological section was at the same location as the MRI slice. Electrophysiological measurements in the internal capsule were done on horizontal slices at the level of the anterior commissure approximately at the same distance from bregma as described above for coronal slices ( $1 \mathrm{~mm}$ posterior). Data were sampled from approximately the same regions of the posterior corpus callosum and internal capsule. The voxel values on MRI, histological counts, and multiple electrophysiological measurements in the selected ROIs on the adjacent slices were averaged.

\section{Statistical analysis}

Data are presented as means \pm SEM. A comparison across ages was done with single-factor ANOVA, followed by Tukey's multiple-comparisons tests or by $t$ test for the regression slopes between age groups, across examined parameters and structures. The differences were considered significant if the $p$ value was $<0.05$. A comparison of three different slopes (with three different units) was done by making the maximum mean value equal to $85 \%$ of the ordinate range, normalizing the curve to the range, adjusting for the decreased rate of change by converting the days to the logarithmic value, performing a linear regression of the normalized percentage of change from E29 with log days, and comparing the slopes by confidence intervals.

The study was approved by the Animal Review Committee of the Evanston Northwestern Healthcare Research Institute. All animals received humane care in compliance with the National Institutes of Health Guide for the Care and Use of Laboratory Animals (publication number 85-23, revised 1985).

\section{Results}

Given that anisotropy is thought to be related to the progression of myelination, we first immunohistochemically defined the timing of appearance of early myelination by $\mathrm{O} 1$ staining and mature myelination by MBP staining and appearance of $\mathrm{NaCh}$ clusters. We also investigated an earlier stage of oligodendrocyte, the preoligodendrocytes, that is defined by $\mathrm{O} 4$ antigen staining (and is $\mathrm{O} 1$ negative).

\section{Immature oligodendrocytes population increases with white-matter development}

The population pool of $\mathrm{O} 4+$ cells in the corpus callosum and internal capsule generally increases with age with some variability (Fig. 2). The number of O1+ cells steadily increases with age (Fig. 2 ) and represents the population of immature oligodendrocytes.

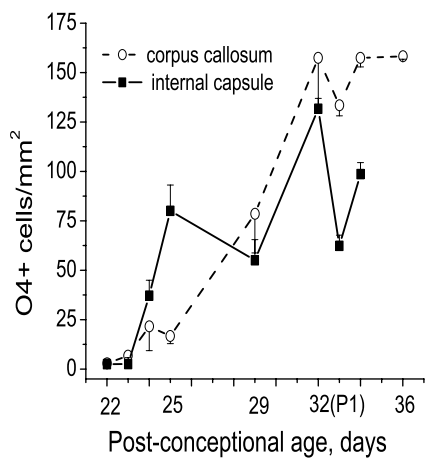

A

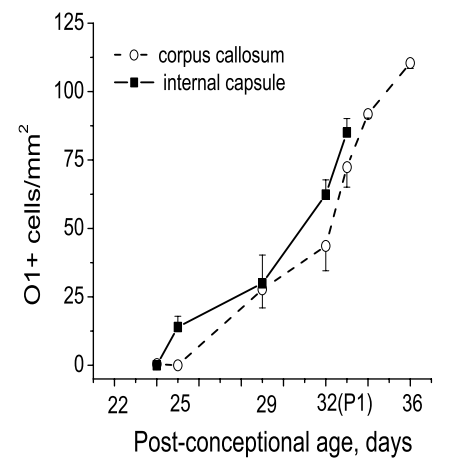

B
Figure 2. Cell counts were obtained from $50-\mu \mathrm{m}$-thick adjacent sections that were alternately stained with the 04 or 01 antibody $\boldsymbol{A}$, The population pool of $04+$ cells in the corpus callosum (open circles and dashed lines) and internal capsule (squares and solid lines) generally increases with age (ANOVA; $p<0.05$ ). $\boldsymbol{B}$, The $01+$ cells exhibit a steady increase with age $(A N O V A ; p<0.05)$. Note the later onset of immature oligodendrocytes in the corpus callosum.

The difference between the $\mathrm{O} 4$ curve and the $\mathrm{O} 1$ curve represents approximately the population of preoligodendrocytes. At P5, because of maturation and development of myelinated fibers, counting of preoligodendrocytes and immature oligodendrocytes becomes technically difficult.

\section{Different timing of myelin maturation for the internal capsule and corpus callosum}

At P5, the appearance of mature myelination is supported by visible $\mathrm{NaCh}$ clustering and $\mathrm{MBP}$ staining. $\mathrm{NaCh}$ clusters and MBP staining appeared at P5 in the internal capsule and cingulum (Fig. $3 A, C$ ) but not in the center of the corpus callosum (Fig. $3 B)$. NaCh clusters increased in number and in density with more intense MBP staining at P11 in the internal capsule (Figs. $3 E, G$ ), cingulum, corona radiata, and anterior commissure (data not shown). Multiple clusters of $\mathrm{NaCh}$ were observed at P11 in these areas. In contrast, single clusters of $\mathrm{NaCh}$ and some $\mathrm{MBP}$ staining only begin to appear in a more ventral location in the center of the corpus callosum at P11 (Fig. $3 F, H$ ). At an earlier postnatal age, $\mathrm{P} 1$, there was no visible $\mathrm{MBP}$ staining or $\mathrm{NaCh}$ clustering at $\mathrm{P} 1$ in the corpus callosum or internal capsule. $\mathrm{NaCh}$ immunolabeling was faint and diffuse in both structures at this time. The advantage of staining for $\mathrm{NaCh}$ clustering was the quantitative assessment of the state of myelination in different white-matter tracts. From P5 to P11, there was a 7- to 10 -fold increase in $\mathrm{NaCh}$ density in the internal capsule and dorsolateral corpus callosum (Fig. 4A). This upsurge was in marked contrast to the very small number of $\mathrm{NaCh}$ clusters in the center of the corpus callosum (Fig. 4A).

\section{CAP changes in white-matter development}

Early functional maturation of white matter was evaluated using electrophysiology, by the assessment of CAP and the CV in selected white-matter tracts, the internal capsule and corpus callosum. The CAP of fetal and neonatal rabbit pups in both measured white-matter structures had monophasic positive and negative components with wide shape (Fig. $1 B$ ), as expected for immature white matter with a slow propagation velocity (Rasband et al., 1999). The amplitude of the positive peak and area of the CAP increased proportionally to the stimulus amplitude, with the peak of the positive component of the CAP response shifting to the right. Saturation levels of the corpus callosum CAP amplitude increased, from $2.4 \pm 1.0 \mathrm{mV}$ in $\mathrm{P} 1$ pups to $3.5 \pm 1.8 \mathrm{mV}$ in P11 

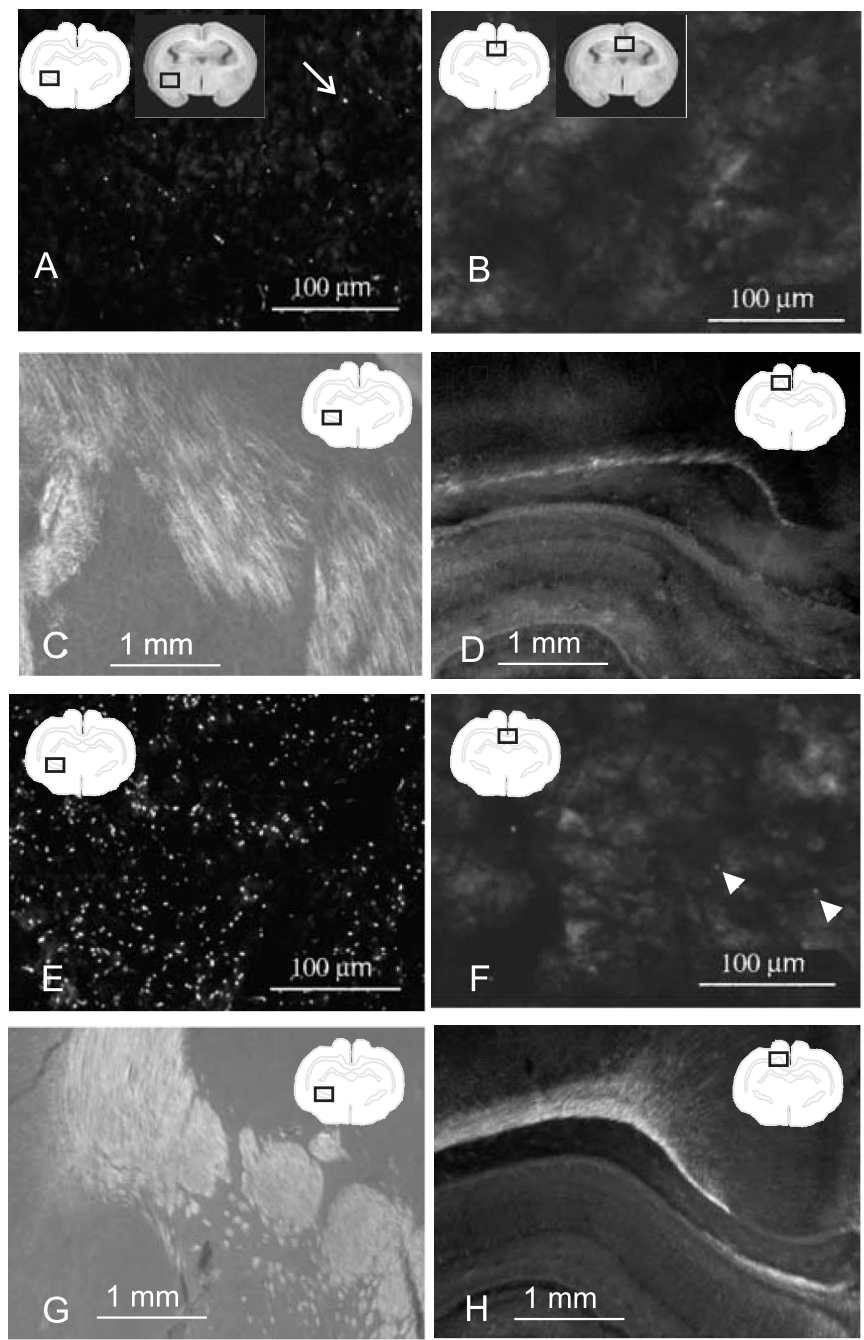

Figure 3. Frozen 5-6 $\mathrm{mm}$ coronal sections of brains were stained with a pan-isoformspecific polyclonal antibody against voltage-gated $\mathrm{NaCh}$ (first and third rows). MBP staining was done on $20 \mu \mathrm{m}$ paraformaldehyde-fixed sections (second and fourth rows). Rectangles on the diagrams and the $\mathrm{T} 2$ images at the panel corners outline the position of the panel on the slice. The internal capsule is shown in $\boldsymbol{A}, \boldsymbol{C}, \boldsymbol{E}$, and $\boldsymbol{G}$; the corpus callosum is shown in $\boldsymbol{B}, \boldsymbol{D}, \boldsymbol{F}$, and $\boldsymbol{H}$. The images are from P5 pups $(\boldsymbol{A}-\boldsymbol{D})$ and P11 pups $(\boldsymbol{E}-\boldsymbol{H})$. There were no visible $\mathrm{NaCh}$ clusters at P1 (data not shown). In $\boldsymbol{B}$ and $\boldsymbol{F}$, there are areas of higher-intensity staining that represent diffuse staining from $\mathrm{NaCh}$ (on higher power). Note that the increased diffuse intensity is not representative of clustering of $\mathrm{NaCh}$. NaCh clusters appear as bright puncta first in the internal capsule ( $\boldsymbol{A}$, arrow) and dorsolateral corpus callosum at P5, along with MBP staining $(\boldsymbol{C}, \boldsymbol{D})$. There was a marked increase in the number of clusters in the internal capsule and dorsolateral corpus callosum at P11. Even at P11, there are very few NaCh clusters (arrowheads) in the center of the corpus callosum $(\boldsymbol{F})$. Each panel has a drawing in the corner, indicating the location on the slice where the images were taken.

pups. The level of stimulation intensity for maximal CAP decreased with age from $6.07 \pm 0.57 \mathrm{~mA}$ at E29 to $3.81 \pm 0.50 \mathrm{~mA}$ at P11. All measurements of CAP area and CV were subsequently made with a stimulation intensity of $6.5 \mathrm{~mA}$, slightly above the level necessary to evoke the maximal CAP. The CAP area also significantly increased with age (ANOVA; $p<0.01$ ) from P1 to P11 (Fig. 1D). The CAP area in the corpus callosum showed an almost linear increase between E29 and P5, 18.5\% per day, with subsequent change of the CAP slowing down to $2.7 \%$ per day from P5 to P11 (comparison of slopes; $t$ test; $p<0.01$ ) (Fig. $4 B$ ), similar to the pattern of changes in FA. The CAP area in the internal capsule had a similar response to that in the corpus callosum, 32.1\% per day from E29 to P5, with slowing between P5 and P11 to $5.1 \%$ per day (comparison of slopes; $t$ test; $p<0.05$ ) (Fig. $4 B$ ).

\section{Differential conduction velocity changes in} white-matter tracts

The CV in the corpus callosum exhibited a rapid increase from E29 to P5, 12.2\% per day. The rate of increase diminished from $\mathrm{P} 5$ to $\mathrm{P} 11$ to $<2 \%$ per day (comparison of slopes; $p<0.01$ ) (Fig. $4 C)$, similar to the changes in CAP area and FA. The CV in the internal capsule, in contrast, continued to show a greater increase between E29-P5 and P5-P11, The rate of increase between P5 and $\mathrm{P} 11$ in the internal capsule, $18.1 \%$ per day, was much greater than that in the corpus callosum $(p<0.01)$. The shape of the CAP recorded in some locations of the internal capsule at $\mathrm{P} 11$ had a biphasic positive component such as that seen in more mature brains. Interestingly, the CV in both structures between E29 and P1 had values typical of nonmyelinated white matter, with slow conduction of $0.2-0.35 \mathrm{~m} / \mathrm{s}$. In comparison, adult rabbits had values of $1.84 \pm 0.43 \mathrm{~m} / \mathrm{s}$ in the corpus callosum and $1.79 \pm 0.82$ $\mathrm{m} / \mathrm{s}$ in the internal capsule, almost 10 times that of the neonatal rabbit.

\section{Perinatal rabbit white-matter development assessed by DTI}

Most major white-matter structures, including the internal capsule, corona radiata, fimbria of the hippocampus, and the stria terminalis, were visible on the FA map by E22. However, the corpus callosum was not visible until E25 (Fig. 5A), consistent with the low density of O4- and O1-labeled cells. From E25 onward, major white-matter structures could be delineated (Fig. $5 B-D)$. Thus, delineation of white matter from gray matter begins before birth in the rabbit. Some white-matter tracts such as the corpus callosum lag behind other tracts. For the purpose of brevity and clarity, we will present findings predominantly from two white-matter structures: the corpus callosum and the internal capsule.

\section{Age-dependent mean diffusivity decrease in perinatal cerebral} white matter

The ADC values for selected gray- and white-matter structures are presented in Table 1. The mean diffusivity or ADC of all white-matter regions significantly decreased with age (Fig. 6A). The fastest rate of change was observed in areas with projection fibers: the internal capsule and corona radiata. There was up to a $20 \%$ decrease in the internal capsule from P1 to P11. The change was less in commissural fibers, such as the corpus callosum (5\%), and in limbic fibers, such as the fornix fimbria (14\%). By P11, the $\mathrm{ADC}$ in white-matter tracts such as the internal capsule reached $1.0 \times 10^{-3} \mathrm{~mm}^{2} / \mathrm{s}$, a value observed in adult cats (Baratti et al., 1999). These changes in the ADC correlated with a decrease in $T 2$ (correlation coefficient, 0.576; $p<0.01$ ) from $125-135 \mathrm{~ms}$ at $\mathrm{P} 1$ to $95-115 \mathrm{~ms}$ at $\mathrm{P} 11$ in white matter (Fig. 6 B). Comparatively, the ADC in white matter was significantly higher than in gray matter at P1, probably reflecting relatively higher water and less cell and membrane content. With age, gray-matter diffusivity also decreased and both gray and white matter reached near adult values by $\mathrm{P} 11$.

\section{Anisotropy increased with age in white matter}

FA values for the examined gray- and white-matter structures are presented in Table 2. The most pronounced change in diffusion properties of the developing rabbit brain was the change in anisotropy of white matter as measured by FA. FA increased significantly with aging in all measured white-matter structures 


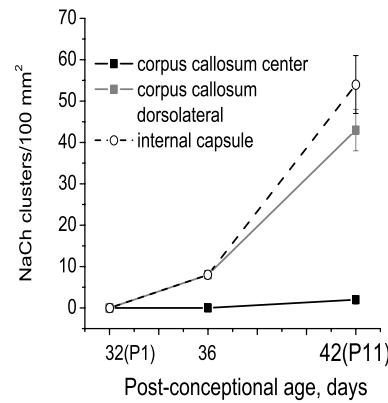

A

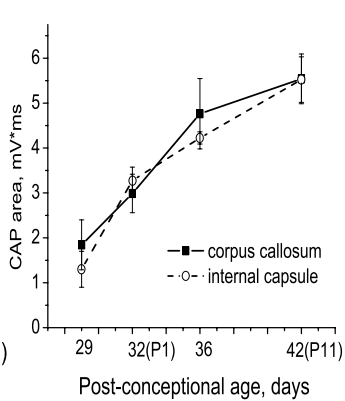

B

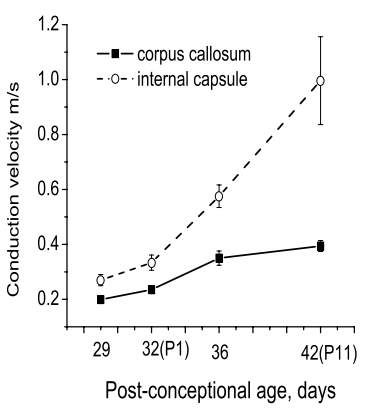

C
Figure 4. A, Frozen brain slices $(6 \mu \mathrm{m})$ were incubated with a pan-isoform-specific monoclonal antibody against voltagegated sodium channels ( $\mathrm{NaCh}$ ) and were then incubated with an HRP-labeled secondary antibody. NaCh clusters were counted per $100 \mu \mathrm{m}$ on six FOVs per slice. There was a fivefold to sixfold increase in NaCh clusters (ordinate) in the internal capsule (IC) compared with the corpus callosum (CC) from P5 to P11, reflecting the contribution of the process of myelination. The center of the $\mathrm{CC}$ remains unmyelinated at $\mathrm{P} 11$. Note that the dorsolateral region of the $\mathrm{C}($ behaves like the $\mathrm{IC}$ with earlier myelination than that in the center of the $\mathrm{CC}$. Measurements of $\mathrm{NaCh}$ clusters were taken on coronal slices at the level of the hippocampus $(1 \mathrm{~mm}$ posterior to bregma). Data are presented as mean \pm SEM ( $n=3$ per age per tract). $\boldsymbol{B}$, The methodology of obtaining the CAP area is given in Figure 1. Measurements from the $C($ were taken on coronal slices at the level of the hippocampus and from the IC on horizonta slices at the level of the anterior commissure and $1 \mathrm{~mm}$ posterior to bregma. Stimulation intensity was fixed to a maximal threshold for P1, and distance between electrodes for the CAP area was between $700 \pm 10 \mu \mathrm{m}$. The CAP area (ordinate) increased significantly with age (abscissa) in both the CC and IC (ANOVA; $p<0.01$ ). C, Calculation of the CV is given in Figure 1. CV (ordinate) increases differently with age (abscissa) in the $\mathrm{CC}$ compared with the $\mathrm{IC}$. In the $\mathrm{CC}$, there is an increase from E29 to P5 and then plateau is reached by $\mathrm{P} 11$. In contrast, there is a continued increase in $\mathrm{CV}$ at $\mathrm{P} 11$, probably reflecting the effect of myelinated fibers. There is an increased variability of $\mathrm{CV}$ in the $\mathrm{IC}$ at $\mathrm{P} 11$, reflecting the contribution of both myelinated and unmyelinated fibers. Myelination and the corresponding increase in $\mathrm{CV}$ occurs later in the CC. Adult values are 10 times that at birth in both tracts. Data are presented as mean \pm SEM $(n=3-5$ per age per tract).
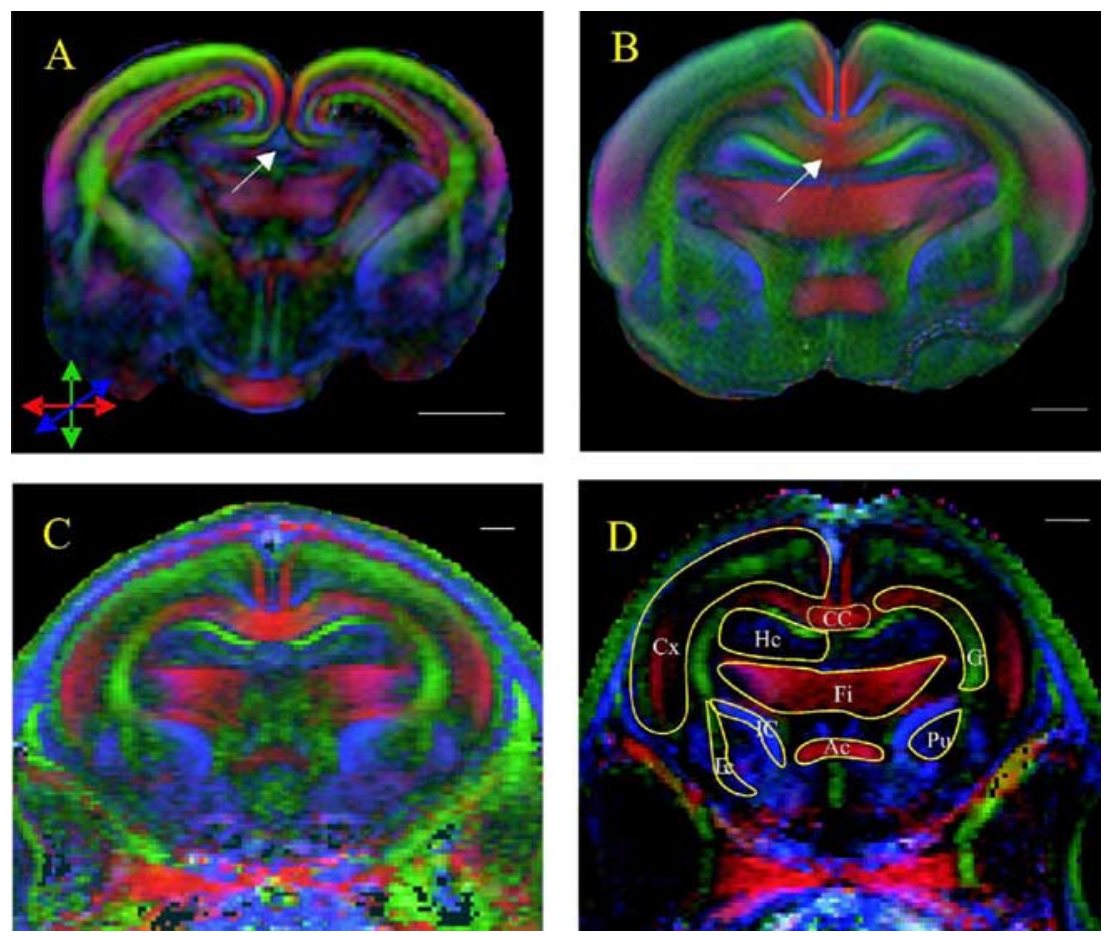

Figure 5. A, A hysterotomy was performed at E22, and fetal brains were extirpated. Brains were immersion fixed with $4 \%$ paraformaldehyde and underwent ex vivo DTI on a 4.7 T magnet: TR/TE, $2500 / 38 \mathrm{~ms} ; b=0$ and $1815 \mathrm{~s} / \mathrm{mm}^{2}$; slice thickness/inplane resolution, $0.5 / 0.165 \mathrm{~mm} ; 12$ averages. Color intensity on the directionally encoded FA map is proportional to the $F A$ value. The predominant diffusion direction is depicted by colored arrows at the bottom left. Most of the white-matter structures are formed by E22, except for the corpus callosum (white arrow). $\boldsymbol{B}$, A similar procedure was done at E25, and the directionally encoded FA map shows the appearance of the corpus callosum (white arrow). C, P11 pup measured in vivo on a 4.7 T magnet: TR/TE, $2000 / 35 \mathrm{~ms} ; b=0$ and $780 \mathrm{~s} / \mathrm{mm}^{2}$; slice thickness/in-plane resolution, $1.5 / 0.195 \mathrm{~mm}$; eight averages. The directionally encoded FA map shows that the volume and anisotropy of white-matter tracts have significantly increased compared with earlier gestation $(\boldsymbol{A}, \boldsymbol{B})$. D, Various white-matter tracts and gray-matter structures were outlined, as shown on the P5 pup map. Automatic segmentation was used to define the corpus callosum (CC) and internal capsule (IC) (see Materials and Methods), and manual segmentation was used for the rest of the structures. Scale bars, $2 \mathrm{~mm}$. Ac, Anterior commissure; Ec, external capsule; Fi, fimbria hippocampi; $\mathrm{Cr}$, corona radiata; $\mathrm{C}$, cerebral cortex; $\mathrm{Pu}$, putamen; $\mathrm{Hc}$, hippocampus.
(ANOVA; $p<0.01$ ) (Fig. 6C). FA increased more than twofold in the corpus callosum and increased by $75 \%$ in the internal capsule from E25 to P11. The rate, magnitude, and timing of FA change were noticeably different between white-matter tracts. The rate of FA increase in the corpus callosum was the highest between E25 and P1 (11.4\% per day; comparison of slopes; $p<0.01)$. From P1 to P5, the rate of increase in FA slowed $(4.1 \%$ per day; $p<0.01)$ and reached a plateau from P5P11 (0.3\% increase per day). In the internal capsule, the highest rate of increase was between E29 and P1 (10.7\% per day; $p<0.01)$. Thereafter, the FA change increased by $<5 \%$ per day. By P11, the FA approached adult values of $0.7-0.8$ in the corpus callosum and $0.65-0.7$ in the internal capsule. Thus, the rapid phase of FA change occurred before the onset of myelination at P5 in the internal capsule and at P11 in the corpus callosum.

To ascertain whether it was feasible to compare anisotropy values before and after birth, we compared FA values obtained from the same P1 brains $(n=4)$ analyzed in vivo and ex vivo (4\% paraformaldehyde fixation). The slice positions were kept approximately the same. The FA between fixed and in vivo samples was not different in either the corpus callosum or internal capsule (paired $t$ test; $p=0.75$ and 0.78 , respectively). The confidence intervals for the difference between fixed and in vivo samples were $0.004-0.050$ for the corpus callosum and -0.052 to -0.030 for the internal capsule. Even if there was a type II error, the possible difference is much smaller than the change from the last fixed sample at E29 to the first in vivo sample at P1. These findings from the developing brain were similar to that observed in adult mouse brains (Sun et al., 2003b). Thus, it was feasible to follow the developmental progression for the change in FA by imaging both fixed fetal brains and live postnatal brains.

\section{Decreased radial diffusivities in white matter}

FA is a directionally invariant index of the diffusion anisotropy and reflects the variance among the three eigenvalues of the diffusion tensor. We next investigated the separate contribution of the directional diffusivities to the growing anisotropy in the white matter in an attempt to better define some of the factors that contribute to fiber tract development. The first eigenvalue of the diffusion tensor is presumed to measure the diffusion rate collinear with white-matter fibers; thus, a measure 
of axial diffusivity. An estimate of radial diffusivity was obtained from the average of the second and third eigenvalues, which reflects diffusion across the fibers in a cylindrical shape. In the postnatal period, axial diffusivity did not change with age in the corpus callosum (Fig. 7A). In contrast, axial diffusivity in the internal capsule decreased significantly with age (ANOVA; $p<$ 0.01) (Fig. 7A), perhaps contributing to the overall decrease in the mean diffusivity in the postnatal brain. Radial diffusivity decreased significantly in all white-matter tracts studied postnatally (ANOVA; $p<0.01$ ), which resulted in a significant elevation of FA (Fig. 7B). The changes in directional diffusivities demonstrate that these two components contribute differently to the elevated FA of the different white-matter tracts. Notably, the change in radial diffusivity in the internal capsule after P5 (onset of myelination) was much slower than before P5 (comparison of slopes between different time intervals; $p<0.01)$.

We also analyzed gray matter anisotropy, although that was not the focus of this study. Cortex anisotropy was high initially at E22 and decreased with age, opposite in direction to white matter (Tables 1, 2 ). Interestingly, anisotropy in the hippocampus followed a different pattern from the rest of the cortex (Table 2) and peaked at E25-E29 ( $p<0.01$; multiple $t$ test with Bonferroni's correction), and the subiculum area was found to be the most anisotropic (Fig. 5B,C).

\section{Increase in immature oligodendrocytes coincides with the CAP and anisotropy} We next conducted post hoc analysis of the trends from analysis of oligodendrocyte lineage progression, electrophysiology, and MR imaging. Because E29 was the earliest time for overlap of all three parameters, we superimposed the parameter changes beginning at E29. The maximum mean value of each curve was depicted as $85 \%$ of the upper limit of the ordinate scale for each curve. The immature oligodendrocyte increase coincided with the increases in the CAP and FA in the corpus callosum (Fig. 8A) and, to a lesser degree, in the internal capsule (Fig. $8 B$ ). Comparison of three different slopes (with three different units) were done by normalizing the curve to the range, adjusting for the decreased rate of change by converting the days to the logarithmic value, performing a linear regression of the normalized percentage of change from E29 with log days, and comparing the slopes by confidence intervals. The slopes from E29 to P5 were not different, as supported by the overlap in the confidence intervals (Fig. 8). The inability to quantify precisely the number of immature oligodendrocytes at P5 coincided with the changes of the CAP and FA reaching a plateau. Slopes from E29 to P11 were not different between the CAP and FA for the corpus callosum (441, $78-804$ vs $480,327-634)$ or the internal capsule $(484,303-665$ vs 434, 265-603; normalized percentage of change/log day; mean, 5 th to 95 th percentiles).

\section{Discussion}

This is the first study to undertake an integrated systemic analysis of cerebral white-matter development in the rabbit. The unique feature of the study are the correlations among oligodendrocyte
Table 1. ADC in white- and gray-matter structures $\left(\times 10^{-3} \mathrm{~mm}^{2} / \mathrm{s}\right)$

\begin{tabular}{lllll}
\hline Postconceptual age & E32 (P1) & E36 (P5) & E39 (P8) & E42 (P11) \\
\hline Fimbria hippocampi & $1.15 \pm 0.05$ & $1.01 \pm 0.03$ & $1.03 \pm 0.05$ & $1.04 \pm 0.02$ \\
Corona radiata & $1.33 \pm 0.04$ & $1.20 \pm 0.03$ & $1.19 \pm 0.02$ & $1.09 \pm 0.05$ \\
Caudate nucleus & $0.98 \pm 0.04$ & $0.89 \pm 0.03$ & $0.93 \pm 0.06$ & $0.95 \pm 0.03$ \\
Cerebral cortex & $1.06 \pm 0.06$ & $0.98 \pm 0.03$ & $1.02 \pm 0.03$ & $0.92 \pm 0.01$ \\
Hippocampus & $1.12 \pm 0.04$ & $1.03 \pm 0.02$ & $1.02 \pm 0.03$ & $1.02 \pm 0.01$ \\
Thalamus & $1.12 \pm 0.04$ & $1.02 \pm 0.04$ & $1.05 \pm 0.02$ & $1.00 \pm 0.03$ \\
\hline
\end{tabular}

The mean diffusivity or $A D C$ of all white-matter regions significantly decreased with age. The $A D C$ of the whitematter structures was significantly higher than that of gray-matter structures at P1. By P11, the ADC values of white matter became closer to those of gray-matter structures.

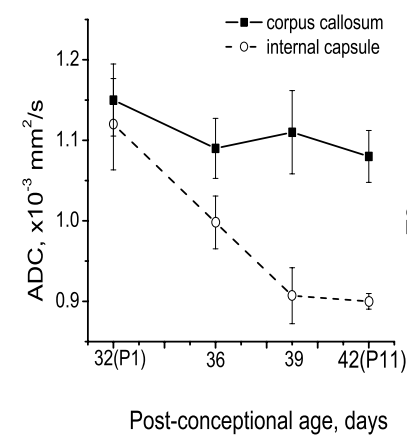

A

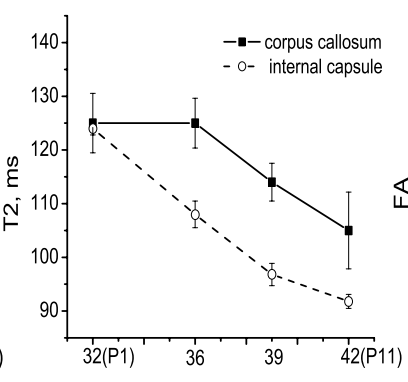

Post-conceptional age, days

B

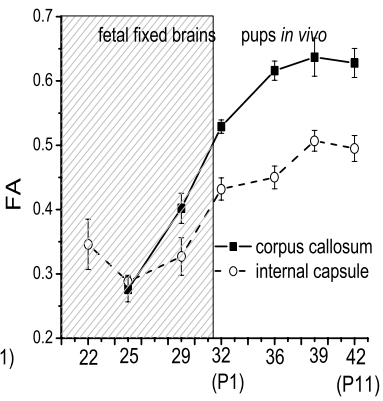

Post-conceptional age, days

C

Figure 6. A, DTl, consisting of a set of diffusion-weighted images in six noncollinear directions, was obtained from live rabbit pups in a $4.7 \mathrm{~T}$ magnet. The ADC of tissue water was calculated as a trace of the diffusion matrix. Postnatally, the ADC decreased $\mathrm{E} 25$ and P5 (E36) for the $\mathrm{CC}$ and between $\mathrm{E} 29$ and P1 for the IC. Subsequently, the rate of increase in FA reaches a plateau. Data are presented as mean \pm SEM $(n=5-9$ per age per tract).

maturation, functional development as measured by electrophysiological techniques, and structural development of whitematter tracts, as measured by DTI. The measurements of CAP, $\mathrm{CV}$, and $\mathrm{NaCh}$ clusters have been used for the first time in $\mathrm{CNS}$ structures.

Around birth, the rabbit brain experiences a period of rapid structural and functional maturational changes. The most rapid change in immature oligodendrocyte density, CAP (both CV and magnitude), FA, and ADC occurs between E29 and P5. After P5, the rate of change decreases, reaching a plateau by $\mathrm{P} 11$. This rapid change occurs before the onset of myelination that starts at P5 for the internal capsule and at P11 for the corpus callosum. The period of rapid maturation suggests that rabbits are perinatal brain developers similar to humans. In humans, myelination of sensory and motor fibers begins at birth (Kinney et al., 1988; Paus et al., 2001) and continues during the first year of life. The fastest rate of change in FA and in the ADC in humans takes place in the first month of life, then slows down and reaches a plateau by the end of the first year (Neil et al., 1998; Mukherjee et al., 2001). Immature oligodendrocytes begin to increase severalfold in the third trimester in human cerebral white matter (Back and Rivkees, 2004). In contrast, rodents are typically postnatal brain developers such that myelination starts later than in rabbits and is more prolonged in duration (Hildebrand et al., 1993). At the opposite end of the spectrum from rodents are sheep, pigs, and monkeys that are prenatal brain developers. By the time of birth, 
Table 2. FA in white- and gray-matter structures

\begin{tabular}{|c|c|c|c|c|c|c|}
\hline Postconceptual age & E22 & E25 & E29 & E32 (P1) & E36 (P5) & E42 (P11) \\
\hline Corpus callosum & & $0.27 \pm 0.02$ & $0.40 \pm 0.02$ & $0.54 \pm 0.02$ & $0.63 \pm 0.01$ & $0.61 \pm 0.04$ \\
\hline Internal capsule & $0.35 \pm 0.04$ & $0.29 \pm 0.09$ & $0.32 \pm 0.03$ & $0.44 \pm 0.01$ & $0.45 \pm 0.02$ & $0.48 \pm 0.02$ \\
\hline Fimbria hippocampi & $0.30 \pm 0.01$ & $0.29 \pm 0.04$ & $0.45 \pm 0.02$ & $0.45 \pm 0.02$ & $0.48 \pm 0.01$ & $0.46 \pm 0.01$ \\
\hline Corona radiata & $0.33 \pm 0.04$ & $0.32 \pm 0.03$ & $0.30 \pm 0.03$ & $0.30 \pm 0.01$ & $0.30 \pm 0.01$ & $0.33 \pm 0.02$ \\
\hline Caudate nucleus & $0.20 \pm 0.02$ & $0.23 \pm 0.04$ & $0.18 \pm 0.02$ & $0.15 \pm 0.01$ & $0.21 \pm 0.02$ & $0.12 \pm 0.01$ \\
\hline Cerebral cortex & $0.32 \pm 0.03$ & $0.32 \pm 0.02$ & $0.33 \pm 0.02$ & $0.26 \pm 0.02$ & $0.23 \pm 0.02$ & $0.20 \pm 0.02$ \\
\hline Hippocampus & $0.20 \pm 0.01$ & $0.28 \pm 0.04$ & $0.31 \pm 0.02$ & $0.21 \pm 0.01$ & $0.20 \pm 0.02$ & $0.14 \pm 0.01$ \\
\hline Thalamus & $0.17 \pm 0.01$ & $0.18 \pm 0.01$ & $0.18 \pm 0.03$ & $0.19 \pm 0.01$ & $0.22 \pm 0.02$ & $0.17 \pm 0.02$ \\
\hline
\end{tabular}

FA in the white and gray matter change in opposite directions with age, with an increase in white matter and a decrease in gray matter. The fastest increase in FA was observed in projection fibers, such as the internal capsule (see Fig. $6 \mathrm{C}$ ) and corona radiata.
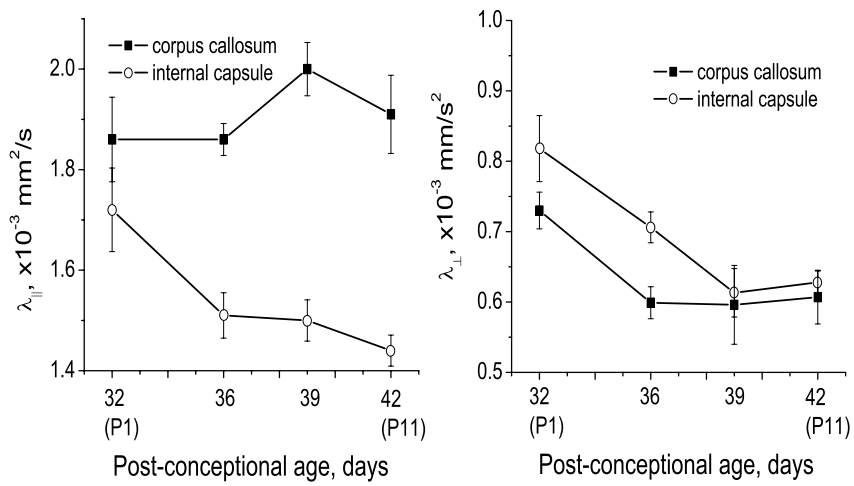

Figure 7. $A$, We measured the directional change in water diffusivity in white-matter tracts. Axial diffusivity ( $\lambda_{\|}$, ordinate), along the course of white-matter tracts, increases with age (abscissa) in the corpus callosum and decreases in the internal capsule. $\boldsymbol{B}$, Radial diffusivity ( $\lambda_{\perp}$, ordinate), across the course of white-matter tracts, decreases with age (abscissa) in both the corpus callosum and internal capsule, resulting in an increase in FA (Fig. 6D). Data are presented as mean \pm SEM $(n=5-9$ per age per tract).

most of their white-matter tracts are myelinated. Thus, structural development of the rabbit brain is close to humans, but with a compressed time frame.

The onset and time course of myelination varies in different white-matter tracts. This is evidenced by the $\mathrm{NaCh}$ clustering and MBP staining that are first observed at P5 for the internal capsule and later at P11 for the corpus callosum. Myelination of commissural and association fibers is known to occur later than projection tracts (Kinney et al., 1988).

Myelination affects diffusion anisotropy differently in different species and white-matter structures. The onset of diffusion anisotropy of the rat optic nerves precedes the earliest histological evidence of myelin in white matter (Wimberger et al., 1995; Prayer et al., 1997) and continues to increase in the cranial nerves in rats from 2 to 10 weeks (Takahashi et al., 2000). In the rat corpus callosum, the extracellular volume fraction starts to decrease at $\mathrm{P} 4$, but the increase in the diffusion anisotropy begins with the onset of myelination at $\mathrm{P} 13$ and does not increase further after reaching a maximum at P17, when myelination is well advanced (Vorisek and Sykova, 1997). Unlike the present study using DTI, these previous studies used two axes-ADC indices or extracellular tracer diffusion, subject to the limitations of inexact orientation. Our study shows that the rate of increase in anisotropy is most rapid before myelination from E25 to $\mathrm{P} 5$ and reaches a plateau at about the time of onset of myelination. Starting at P5, the rate of change slows and reaches a plateau in FA, axial diffusivity, or radial diffusivity. Despite the major concomitant increase in anisotropy after the onset of myelination, it is now believed that myelination is not required for "significant" diffusion anisotropy, based on the high anisotropy observed in non-

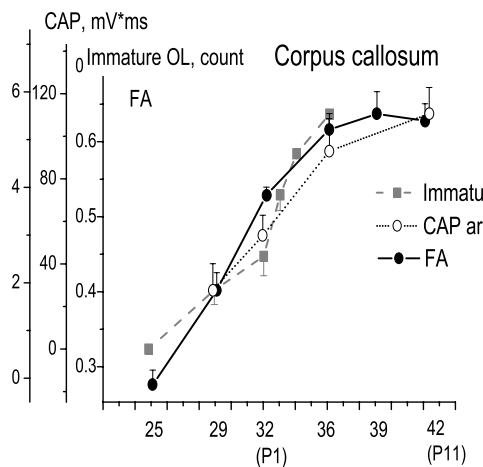

Post-conceptional age, days

A

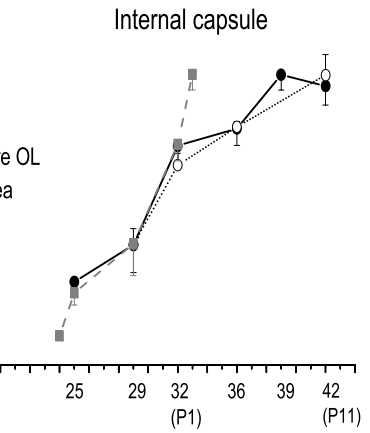

Post-conceptional age, days
Figure 8. $A, B$, Coincidental increase in cells, electrophysiology, and MRI parameters in the corpus callosum $(\boldsymbol{A})$ and internal capsule $(\boldsymbol{B})$. Because E29 was the earliest time point measured for all three parameters, curves are superimposed using E29 as a common starting point. The $y$-axis was adjusted so that the maximum mean value was always $85 \%$ of the maximum ordinate value. The increase in immature oligodendrocytes ( $0 \mathrm{~L}$; gray squares and gray dashed line) coincided with the increase in the CAP (open circles and dotted line) and FA (filled circles and solid lines) in the corpus callosum ( $\boldsymbol{A}$ ) and, to a lesser degree, in the internal capsule $(\boldsymbol{B})$. Note that $01+$ cells cannot be detected at P5 in the internal capsule and at P11 in the corpus callosum as immature oligodendrocytes mature into a myelinating phenotype. Comparison of three different slopes (with three different units) were done by normalizing the curve to the range of values, adjusting for the decreased rate of change by converting the days to the logarithmic value, performing a linear regression of the normalized percentage of change from E29 with log days and comparing the slopes by confidence intervals. Slopes of FA $(803,605-$ 1001) and CAP (797, - 92 to 1685) were not different from that of 01 + cells $(957,732-1182$ normalized percentage of change per log day; mean, 5 th to 95 th percentiles) between $\mathrm{E} 29$ and P5 for the corpus callosum. The abscissa is the same for $\boldsymbol{A}$ and $\boldsymbol{B}$ (postconceptional age).

myelinated fibers in many species and myelin-deficient mutations (Beaulieu, 2002). The fact that additional myelination may not contribute as much to the anisotropy of the white-matter tracts may lie in the fact that a single membrane may present a sufficient barrier for water diffusion.

Various factors are believed to restrict diffusion of water transverse to the axons and make the diffusion anisotropic. With increasing age, diffusion anisotropy is linked to neurofilament development, axon alignment, a growing number of membrane barriers, greater cohesiveness and compactness of the fiber tracts, reduced extra-axonal space (i.e., greater packing), changes in extracellular and intracellular matrix, and, finally, myelin formation as the white matter matures over time (Beaulieu, 2002). The decrease in radial diffusion suggests that packing of fibers may contribute to the increased FA before myelin formation. The increase in axial diffusivity with postnatal age is somewhat harder to explain and may be attributable to more active transport facilitated by oligodendrocytes or attributable to streamlining of fi- 
bers, as proposed recently. An increase in the axial diffusivity in the rat optic nerve at 2-10 weeks was attributed to the streamlining observed on electron micrographs of white-matter fibers with age (Takahashi et al., 2000). Therefore, white-matter diffusion in perinatal rabbits appears to be more affected by increased directionality and compactness in organization of white-matter tracts and less by myelination. Our results of an age-dependent increase in axial diffusivity and decreased radial diffusivity are consistent with the cellular changes during white-matter maturation. However, the relative contribution from each of the brain microstructural factors mentioned above to directional diffusivity requires additional exploration.

It has been suggested that magnitude and area under the CAP curve may serve as quantitative indices of the health of the whitematter tissue (Tekkok and Goldberg, 2001; Baker et al., 2002). The CAP amplitude and area may reflect neuronal excitability, axonal density, and involvement of fibers of different types and sizes. We found that there was an age-related increase in the CAP area in both the corpus callosum and internal capsule, and the rate of increase followed the changes of FA and density of immature oligodendrocytes. The area of a monophasic CAP may thus reflect the contributions of premyelinated fibers. Before myelination, CV coincides with the CAP, FA, and immature oligodendrocytes, but after the onset of myelination, CV coincides with the increase in $\mathrm{NaCh}$ clusters. The rate of rise in CV after P5 in the internal capsule and the fact that adult rabbits have a severalfold higher CV than at birth suggests that CV may be better correlated with the nodes of Ranvier and an ensuing saltatory propagation of the action potential than with anisotropy or oligodendrocytes after myelination. There was also a higher variation in the CV of the internal capsule at P11 (E42), corresponding to the transitional period of active functional maturation. Myelination of fibers begins unevenly and may explain the markedly different values of $\mathrm{CV}$ recorded in adjacent fibers. The $\mathrm{CV}$ of the corpus callosum showed much less variation and followed the CAP area trend, consistent with later myelination of this structure compared with the internal capsule. Postnatally in cats, maturation of the nodes of Ranvier occurs at a time of unremarkable growth in fiber diameter and myelin sheath, implicating the importance of functional maturation of white matter (Berthold and Nilsson, 2002). The functional changes measured by electrophysiology could be explained by axon diameter growth and decrease in extracellular space (Vorisek and Sykova, 1997) that contribute to diffusion anisotropy after myelination, but our study suggests that other factors, such as oligodendrocyte maturation, may also play a role. A rise in the $\mathrm{CV}$ occurs along with myelination $\sim 15-25 \mathrm{~d}$ in mice (Rasband et al., 1999). To show a definite correlation between $\mathrm{CV}$ and myelination would require a more extensive study after myelination, which was beyond the scope of the present study.

Preoligodendrocytes increase rapidly with age from E22. There appear to be two peaks of proliferation, but slopes were not significantly different in different time periods. In contrast to immature oligodendrocyte density, changes in preoligodendrocyte density do not appear to correlate with the rapid increase in FA and CAP. During early myelination, when the somata of immature oligodendrocytes are difficult to detect, the curves of FA and CAP also start to plateau. It is possible that immature oligodendrocytes may actually drive the microstructural changes responsible for FA and axial and radial diffusivity perinatal dynamics. Oligodendrocytes are known to play a crucial role in the formation of $\mathrm{NaCh}$ clusters (Butt et al., 1999).

The number of $\mathrm{NaCh}$ clusters is a reflection of the number of nodes of Ranvier and thus a rough index of the functional axonal development during myelination. This index provides a definite quantitative measure of myelination around the time of its onset and progression. The development of $\mathrm{NaCh}$ clusters shows a similar pattern to $\mathrm{CV}$ with age reflecting the process of ongoing myelination.

In conclusion, this study shows that maturation of diffusion anisotropy across fetal and perinatal development coincides with the expansion of premyelinating immature oligodendrocytes as well as the CAP area. CV changes were more dependent on the progression of myelination and correlated with $\mathrm{NaCh}$ clustering. It now appears feasible to establish the clinical relevance of these findings by the investigation of perinatal white-matter lesions. A prediction of depletion of immature oligodendrocytes in such lesions disrupting the microstructural development of cerebral white-matter tracts, as defined by DTI, can thus be tested.

\section{References}

Back SA, Rivkees SA (2004) Emerging concepts in periventricular white matter injury. Semin Perinatol 28:405-414.

Back SA, Han BH, Luo NL, Chricton CA, Xanthoudakis S, Tam J, Arvin KL, Holtzman DM (2002) Selective vulnerability of late oligodendrocyte progenitors to hypoxia-ischemia. J Neurosci 22:455-463.

Baker AJ, Phan N, Moulton RJ, Fehlings MG, Yucel Y, Zhao M, Liu E, Tian GF (2002) Attenuation of the electrophysiological function of the corpus callosum after fluid percussion injury in the rat. J Neurotrauma 19:587-599.

Baratti C, Barnett AS, Pierpaoli C (1999) Comparative MR imaging study of brain maturation in kittens with $\mathrm{T} 1, \mathrm{~T} 2$, and the trace of the diffusion tensor. Radiology 210:133-142.

Basser PJ, Jones DK (2002) Diffusion-tensor MRI: theory, experimental design and data analysis-a technical review. NMR Biomed 15:456-467.

Basser PJ, Mattiello J, LeBihan D (1994) Estimation of the effective selfdiffusion tensor from the NMR spin echo. J Magn Reson B 103:247-254.

Beaulieu C (2002) The basis of anisotropic water diffusion in the nervous system-a technical review. NMR Biomed 15:435-455.

Berthold CH, Nilsson RI (2002) De- and remyelination in spinal roots during normal perinatal development in the cat: a brief summary of structural observations and a conceptual hypothesis. J Anat 200:391-403.

Butt AM, Duncan A, Hornby MF, Kirvell SL, Hunter A, Levine JM, Berry M (1999) Cells expressing the NG2 antigen contact nodes of Ranvier in adult CNS white matter. Glia 26:84-91.

Cabanes C, Lopez de Armentia M, Viana F, Belmonte C (2002) Postnatal changes in membrane properties of mice trigeminal ganglion neurons. J Neurophysiol 87:2398-2407.

Dammann O, Hagberg H, Leviton A (2001) Is periventricular leukomalacia an axonopathy as well as an oligopathy? Pediatr Res 49:453-457.

Derrick M, Luo NL, Bregman JC, Jilling T, Ji X, Fisher K, Gladson CL, Beardsley DJ, Murdoch G, Back SA, Tan S (2004) Preterm fetal hypoxiaischemia causes hypertonia and motor deficits in the neonatal rabbit: a model for human cerebral palsy? J Neurosci 24:24-34.

Hildebrand C, Remahl S, Persson H, Bjartmar C (1993) Myelinated nerve fibres in the CNS. Prog Neurobiol 40:319-384.

Huppi PS, Inder TE (2001) Magnetic resonance techniques in the evaluation of the perinatal brain: recent advances and future directions. Semin Neonatol 6:195-210.

Kinney HC, Brody BA, Kloman AS, Gilles FH (1988) Sequence of central nervous system myelination in human infancy. II. Patterns of myelination in autopsied infants. J Neuropathol Exp Neurol 47:217-234.

Klingberg T, Vaidya CJ, Gabrieli JD, Moseley ME, Hedehus M (1999) Myelination and organization of the frontal white matter in children: a diffusion tensor MRI study. NeuroReport 10:2817-2821.

Klose U, Mader I, Unrath A, Erb M, Grodd W (2004) Directional correlation in white matter tracks of the human brain. J Magn Reson Imaging 20:25-30.

Miller SP, Vigneron DB, Henry RG, Bohland MA, Ceppi-Cozzio C, Hoffman C, Newton N, Partridge JC, Ferriero DM, Barkovich AJ (2002) Serial quantitative diffusion tensor MRI of the premature brain: development in newborns with and without injury. J Magn Reson Imaging 16:621-632.

Mirmiran M, Barnes PD, Keller K, Constantinou JC, Fleisher BE, Hintz SR, 
Ariagno RL (2004) Neonatal brain magnetic resonance imaging before discharge is better than serial cranial ultrasound in predicting cerebral palsy in very low birth weight preterm infants. Pediatrics 114:992-998.

Mori S, Itoh R, Zhang J, Kaufmann WE, van Zijl PC, Solaiyappan M, Yarowsky P (2001) Diffusion tensor imaging of the developing mouse brain. Magn Reson Med 46:18-23.

Mukherjee P, Miller JH, Shimony JS, Conturo TE, Lee BC, Almli CR, McKinstry RC (2001) Normal brain maturation during childhood: developmental trends characterized with diffusion-tensor MR imaging. Radiology 221:349-358.

Neil J, Shiran S, McKinstry R, Schefft G, Snyder A, Almli C, Akbudak E, Aronovitz J, Miller J, Lee B, Conturo T (1998) Normal brain in human newborns: apparent diffusion coefficient and diffusion anisotropy measured by using diffusion tensor MR imaging. Radiology 209:57-66.

Pajevic S, Pierpaoli C (1999) Color schemes to represent the orientation of anisotropic tissues from diffusion tensor data: application to white matter fiber tract mapping in the human brain. Magn Reson Med 42:526-540.

Paus T, Collins DL, Evans AC, Leonard G, Pike B, Zijdenbos A (2001) Maturation of white matter in the human brain: a review of magnetic resonance studies. Brain Res Bull 54:255-266.

Prayer D, Roberts T, Barkovich AJ, Prayer L, Kucharczyk J, Moseley M, Arieff A (1997) Diffusion-weighted MRI of myelination in the rat brain following treatment with gonadal hormones. Neuroradiology 39:320-325.

Prayer D, Barkovich AJ, Kirschner DA, Prayer LM, Roberts TP, Kucharczyk J, Moseley ME (2001) Visualization of nonstructural changes in early white matter development on diffusion-weighted MR images: evidence supporting premyelination anisotropy. AJNR Am J Neuroradiol 22:1572-1576.
Rasband MN, Peles E, Trimmer JS, Levinson SR, Lux SE, Shrager P (1999) Dependence of nodal sodium channel clustering on paranodal axoglial contact in the developing CNS. J Neurosci 19:7516-7528.

Song SK, Sun SW, Ramsbottom MJ, Chang C, Russell J, Cross AH (2002) Dysmyelination revealed through MRI as increased radial (but unchanged axial) diffusion of water. NeuroImage 17:1429-1436.

Sun SW, Song SK, Hong CY, Chu WC, Chang C (2003a) Directional correlation characterization and classification of white matter tracts. Magn Reson Med 49:271-275.

Sun SW, Neil JJ, Song SK (2003b) Relative indices of water diffusion anisotropy are equivalent in live and formalin-fixed mouse brains. Magn Reson Med 50:743-748.

Takahashi M, Ono J, Harada K, Maeda M, Hackney DB (2000) Diffusional anisotropy in cranial nerves with maturation: quantitative evaluation with diffusion MR imaging in rats. Radiology 216:881-885.

Tekkok SB, Goldberg MP (2001) Ampa/kainate receptor activation mediates hypoxic oligodendrocyte death and axonal injury in cerebral white matter. J Neurosci 21:4237-4248.

Volpe JJ (2001) Neurobiology of periventricular leukomalacia in the premature infant. Pediatr Res 50:553-562.

Vorisek I, Sykova E (1997) Evolution of anisotropic diffusion in the developing rat corpus callosum. J Neurophysiol 78:912-919.

Wimberger DM, Roberts TP, Barkovich AJ, Prayer LM, Moseley ME, Kucharczyk J (1995) Identification of "premyelination" by diffusion-weighted MRI. J Comput Assist Tomogr 19:28-33.

Zhang J, Richards LJ, Yarowsky P, Huang H, van Zijl PC, Mori S (2003) Three-dimensional anatomical characterization of the developing mouse brain by diffusion tensor microimaging. NeuroImage 20:1639-1648. 